\title{
Monitoring the Depth of Anesthesia and Current Technology
}

\author{
Daniel Alfonso Botero-Rosas* and Oscar Leonardo Mosquera-Dussán \\ Universidad de La Sabana, Colombia
}

Submission: February 05, 2017; Published: March 06, 2017

*Corresponding author: Daniel Alfonso Botero-Rosas, Grupo PROSEIM, Doctorado en Biociencias, Universidad de La Sabana, Chía, Colombia, Email: daniel.botero@unisabana.edu.co

Abbreviations: GA: General Anesthesia; EEG: Electroencephalographic Signal; SE: State Entropy; RE: Response Entropy; BIS: Bispectral index

\section{Introduction}

General anesthesia (GA) is defined as a drug-induced loss of consciousness during which patients are not arousable, even by painful stimulation [1]. GA takes an important role in surgical procedures where an anesthetic overdose may lead to drug-associated toxicities, coma and even death; on the other hand a light anesthetic dose may lead to the well-known event of intraoperative awareness, which can cause sleep disorders, depression, night terrors, hospitals fears and post-traumatic stress disorder [2-4]. In this context, monitoring depth of anesthesia has become an important issue in anesthesiology.

Electroencephalographic signal (EEG) reflects the activity of the central nervous system and it has been widely used for monitoring depth of anesthesia. In general terms, the EEG of an anesthetized patient changes from high frequency, low amplitude when awake to low frequency, high amplitude when anesthetized; it is also noted that, during the anesthesia procedure the degree of EEG disorder is reduced. Therefore, the concept of entropy was introduced in EEG signal processing. Entropy is related to the complexity of a signal, and has been considered a promising measure of states of consciousness [5]. State Entropy (SE) and Response Entropy (RE) are indices provided by Datex-Ohmeda $\mathrm{S} / 5^{\mathrm{TM}}$ entropy module (General Electric, Finland), which is currently a reference in EEG monitoring during general anesthesia [6,7]. SE and RE are based on spectral entropy computation over the Fourier spectrum; a description of the algorithm applied is available elsewhere [8]. The M-Entropy module is considered a reference in monitoring the depth of anesthesia based on EEG analysis, particularly the Response Entropy (RE) index, was considered a better predictor of patient response to painful stimuli than the Bispectral index (BIS) [9]
Brain electrical activity indices provide an a dimensional number. Generally, a value between 40 and 60 is associated with an appropriate depth of anesthesia, higher values are associated with an awake patient and lower values with a very deep anesthesia. Although this information is useful, it should be used carefully, there could be cases in which the monitor may provide a paradoxical behavior, the next section introduce a report of a paradoxical cases identified at Clínica Universidad de La Sabana when using the Datex-Ohmeda indices.

\section{EEG monitor and paradoxical behavior}

There was a case in which the current technology (SE, RE) provide a parodoxical behaviour that did not match with the clinical assesment. A 49 years-old female patient, with procedure hysteroscopy and endometrial biopsy. In this case SE and RE show high values associated with an awake patient during the surgery (Figure 1).

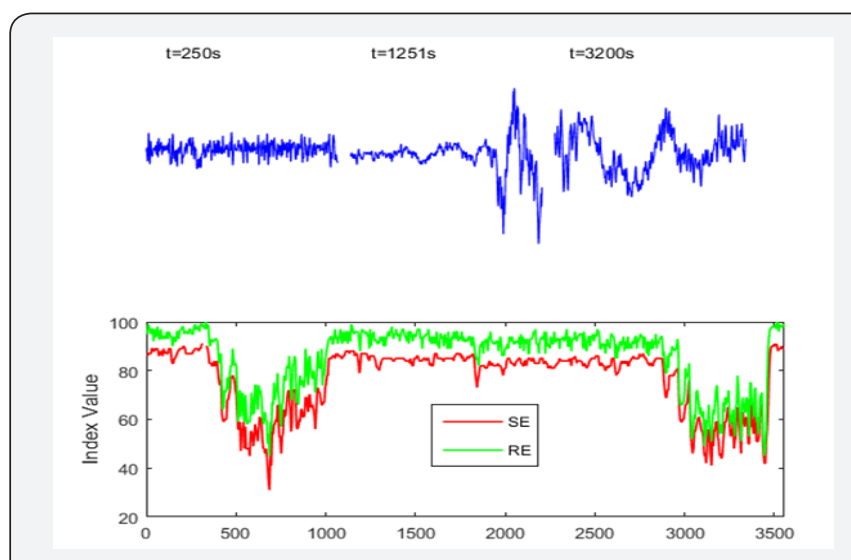

Figure 1: Paradoxical behavior of SE and RE. Upper graph shows a segment of the EEG wave forms; lower graph, shows the Datex-Ohmeda Indices SE (red) and SE (green). 
Automatic impedance test from Datex-Ohmeda monitor showed no problem; this paradoxical behavior alerts the anesthesiology to increase doses of propofol from 2.5 to 3.0 $\mu \mathrm{g} . \mathrm{ml}^{-1}$ and remifentanyl from 5.0 up to $7.0 \mathrm{ng} \cdot \mathrm{ml}-1$ at $1080 \mathrm{~s}$. After three minutes of the same paradoxical behavior, Datexohmeda indices were still inconsistent with the apparent state of the patient. The clinician decided to discarded SE and RE indices information for the rest of the procedure and based the decisions on standard monitoring and clinical assessment. Patient was followed-up by a phone interview 3 and 10 days after the procedure, no sign of dreams, intraoperative awareness or recall was reported.

A possible explanation for the paradoxical behaviour of Datexohmeda indices could be a failure to detect the interspersed low amplitude with high amplitude $E E G$ pattern observed in upper graph of Figure 1, this pattern could be misinterpreted as a contaminated awake EEG resulting in a paradoxical increase in SE and RE, it can be observed that the signal reveals the burst supression pattern. Datex ohmeda indices return to a value associated with clinical state of the patient (40-60) 350s after the TCI was suspended at 2670 s, and the EEG alpha spindles pattern becomes more clear.

\section{Conclusion}

In conclusion, there are cases in which current technology could misinterpreted EEG patterns. If the anesthesiologists are not aware of this situation, is likely that they deepen what is already deep anesthesia.It is important to realize that unexpectedly high quantitative EEG indices values are relatively common and may result in dangerous anesthetic drug overdose [10].

\section{References}

1. Apfelbaum JL, Arens JF, Cole DJ, Connis RT, Domino KB, et al. (2006) Practice advisory for intraoperative awareness and brain function monitoring: a report by the american society of anesthesiologists task force on intraoperative awareness. Anesthesiology 104(4): 847-864.

2. Osterman JE, Hopper J, Heran WJ, Keane TM, van der Kolk B (2001) Awareness under anesthesia and the development of posttraumatic stress disorder. Gen Hosp Psychiatry 23(4): 198-204.

3. Errando CL, Aldecoa C (2014) Awareness with explicit recall during general anaesthesia: Current status and issues. Br J Anaesth 112(1): $1-4$.

4. Sebel PS, Bowdle TA, Ghoneim MM, Rampil IJ, Padilla RE, et al. (2004) The incidence of awareness during anesthesia: A multicenter United States study. Anesth Analg 99: 833-839.

5. Bein B. Entropy. Best Pract Res Clin Anaesthesiol. 2006;20(1):101-9.

6. Schmidt GN, Bischoff P, Standl T, Hellstern A, Teuber O, et al. (2004) Comparative evaluation of the Datex-Ohmeda S/5 Entropy Module and the Bispectral Index monitor during propofol-remifentanil anesthesia. Anesthesiology 101(6): 1283-1290.

7. Ellerkmann RK, Liermann M, Alves T, Wenningmann I, Kreuer S, et al. (2004) Spectral Entropy and bispectral index as measures of the EEG effects of sevoflurane. Anesthesiology 101: 1275-1282.

8. Viertiö-Oja H, Maja V, Särkelä M, Talja P, Tenkanen N, et al. (2004) Description of the EntropyTM algorithm as applied in the DatexOhmeda S/5TM Entropy Module. Acta Anaesthesiol Scand 48(2): 154161.

9. Wheeler P, Hoffman WE, Baughman VL, Koenig H (2005) Response entropy increases during painful stimulation. J Neurosurg Anesthesiol 17(2): 86-90.

10. Bennett C, Voss LJ, Barnard JPM, Sleigh JW (2009) Practical use of the raw electroencephalogram waveform during general anesthesia: The art and science. Anesth Analg 109(2): 539-550.

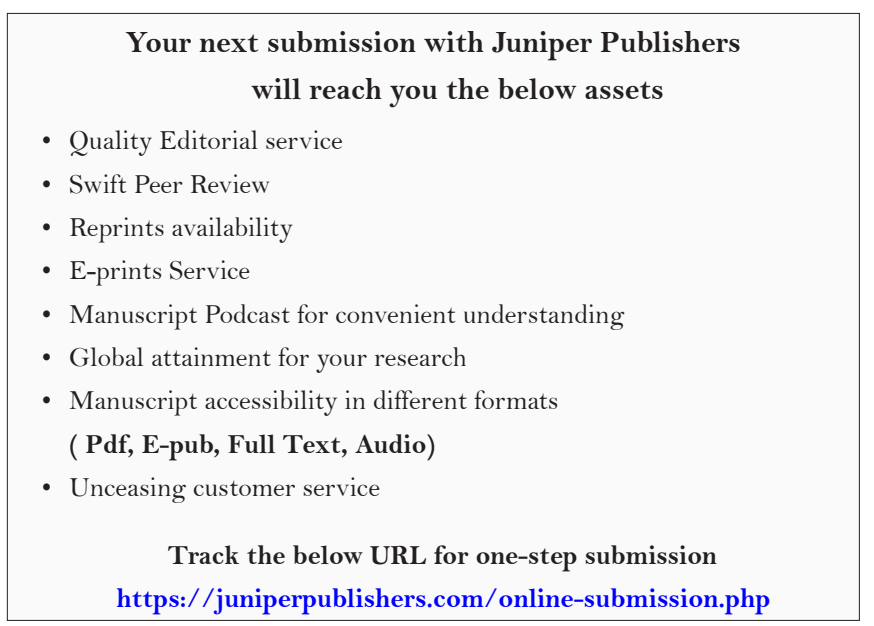

